Research Article

\title{
Opinion of doctors about unmet needs of rural diabetics
}

\author{
${ }^{1}$ Department of Pharmacology, \\ Navodaya Medical College, \\ Raichur, India \\ ${ }^{2}$ Department of Pharmacology, \\ Mamata Medical College, \\ Khammam, India
}

Received: 20 October 2015

Revised: 15 November 2015

Accepted: 17 December 2015

*Correspondence to:

Dr. Rohit Dixit,

Email: rohitdixit.mbbsmd @ gmail.com

Copyright: (C) the author(s), publisher and licensee Medip Academy. This is an open-access article distributed under the terms of the Creative Commons Attribution Non-Commercial License, which permits unrestricted non-commercial use, distribution, and reproduction in any medium, provided the original work is properly cited.

\author{
Rohit Dixit $^{1 *}$, Naveen Pokala ${ }^{2}$
}

\section{ABSTRACT}

Background: India is home to nearly 62 million diabetics - second only to China which has over 92 million diabetics. The main challenges for screening for diabetes and its complications in rural India are inadequate health care resources, lack of awareness and illiteracy among patients, limited physicians and paramedical staff trained in diabetes, lack of awareness among doctors as to who can legally practice diabetes in India. The present study was undertaken to explore the opinion of various doctors regarding diabetic care in rural India.

Methods: The present study was conducted in Navodaya Medical College, Raichur and Mamata Medical College, Khammam. A cross-sectional study design was used to perform a multicentre survey from April 2015 to September 2015. Descriptive analysis was done using Statistical Package for Social Sciences (SPSS). Content and construct validation of the questionnaire was done by a panel containing subject experts, epidemiologists and a statistician. Following which the questionnaires were sent to different set doctors by print outs, email and via online survey tools.

Results: All the doctors who were involved in the study had different opinion. MBBS doctors are more aware than BHMS/BAMS/BUMS doctors about the minimum degree required doing a diabetic practice legally in India. Both the group of doctors believed that the needs of diabetic population in rural India are not properly addressed; the reason behind this was lack of qualified doctors (according to majority of BHMS/BAMS/BUMS doctors) and lack of awareness among the rural patients (according to MBBS doctors). Both the doctors believe that MBBS doctors with some fellowship courses in diabetes can manage diabetes effectively (except serious complications).

Conclusions: Both the doctors believe that MBBS doctors with some fellowship courses in diabetes can manage diabetes effectively (except serious complications). In India only MD (general medicine) and endocrinologists are technically qualified to treat diabetes. MCI should come forward and recognize the fellowship courses by regularizing them with proper guidelines and protocol set up for the institution providing such courses and allow those doctors to practice in rural areas, so that diabetics' needs are addressed properly.

Keywords: Primary care, Prevention, Survey, Sugar, Complications, MCI

\section{INTRODUCTION}

Diabetes mellitus is a group of metabolic diseases characterized by hyperglycemia resulting from defects in insulin secretion, insulin action, or both. Diabetes is a serious and increasing global health burden. According to a recent estimate in 2013, the prevalence of diabetes globally is around 382 million; this number is expected to rise to 592 million by 2035 . Most people with diabetes live in low- and middle-income countries and these will experience the greatest increase in cases of diabetes over the next 22 years. ${ }^{1}$ A diabetes tsunami is headed our way, say experts. One in three adults and nearly one in two men already have prediabetes. The chief culprits: twothirds of adults (and one-third of children) are overweight or obese, and we're a nation of couch potatoes (and computer, car, TV, and phone).

\section{Disease burden (Indian scenario)}

India is home to nearly 62 million diabetics - second only to China which has over 92 million diabetics. By 2030, India's diabetes numbers are expected to cross the 100 million mark according to a 2012 report by International Diabetes Federation. ${ }^{2}$ Uncontrolled diabetes leads to both short term and long term complications which can be 
grouped into microvascular (due to damage to small blood vessels) and macrovascular (due to damage to larger blood vessels). Microvascular complications include damage to eyes (retinopathy) leading to blindness, to kidneys (nephropathy) leading to renal failure and to nerves (neuropathy) leading to impotence and diabetic foot disorders (which include severe infections leading to amputation). ${ }^{3}$ With all these complications the cost of health care to this population is very high. The economic burden due to diabetes in India is among the highest in the world. ${ }^{4}$ The average annual expenditure of those who attend the specialty centre is quite high. The expenditure increases if the patients are hospitalized and further rise will be incurred if they need any surgery. Patients with diabetic complication such as foot complications or kidney complication require additional and continuing expenses for specialized care. A study has estimated the annual cost could be Rs. 90,200/- million (U.S. \$ 2.2 billion) for diabetes health care in India (considering a rather low prevalence of 20 million diabetic patients in India). ${ }^{5}$

\section{Interventions}

It has been suggested by experts that with the correct treatment and recommended dietary and lifestyle changes, many people with prediabetes / diabetes are able to prevent or delay the development of diabetes and its complications respectively. ${ }^{6}$ Many organizations, associations, health care experts have their own set of guidelines and recommendations. Strategies include primary prevention to prevent diabetes, secondary prevention to prevent complications and tertiary prevention to prevent morbidity and mortality. It is, therefore, important to intervene at all levels of diabetes prevention. $^{7}$

\section{Diabetes an iceberg disease}

Individuals who are known to have diabetes represent the tip of the iceberg as an equal or even larger number of patients has undiagnosed diabetes. They are at an even greater risk of adverse clinical outcomes as they are unaware of their condition. ${ }^{8}$ International Diabetes Federation (IDF) estimated that $33 \%$ of adults with diabetes in India are undiagnosed, preventing proper management of the disease by health care team. ${ }^{9}$

\section{Current problem status in rural India}

Although the prevalence of diabetes is lower in rural India, the total number of diabetic subjects in rural areas is much higher, since over $70 \%$ of the population in India lives in rural areas. ${ }^{10}$ Now a day, the rural diabetic population is also on rise. In one study conducted in Pune, for every 100 people surveyed there were at least nine people who were diabetic. ${ }^{11}$ Another study estimates southern cities having a higher prevalence like Trivandrum (9.2\%); Chennai $(6.4 \%)^{12}$ With such prevalence pattern, rural population remains exposed to high level of blood sugar for long time due to lack of screening facility of diabetes at PHC level, and this increases the chance of developing various complication of diabetes mellitus. The main challenges for screening for diabetes and its complications in rural India are inadequate health care resources, lack of awareness and illiteracy, limited physicians and paramedical staff trained in diabetes. ${ }^{13}$ These physicians and paramedical staff can be trained properly so that they can screen and manage diabetes and its complications at the primary care level and refer timely to a tertiary centre.

The present study is done with the aim to explore the opinion of various doctors regarding diabetic care in rural India.

\section{METHODS}

The present study was conducted in Navodaya Medical College, Raichur and Mamata Medical College, Khammam. A cross-sectional study design was used to perform a multicentre survey from April 2015 to September 2015. Descriptive analysis was done using Statistical Package for Social Sciences (SPSS). Development of the survey tool: Content and construct validation of the questionnaire was done by a panel containing subject experts, epidemiologists and a statistician. We had also included feedback from various doctors before finalizing the questionnaire. Following which the questionnaires were sent to different set doctors by print outs, email and via online survey tools (Figure 1).

\section{RESULTS}

A total of 350 responses were collected from as many doctors as possible from all the faculties. Among those respondents, 160 were BHMS/BAMS/BUMS doctors and the remaining 190 were Doctors with MBBS degree. The responses to various questions were recorded separately for BHMS/BAMS/BUMS doctors and MBBS doctors and analysed. The following were the questions asked and responses recorded.

When asked regarding the minimum degree required legally to do a diabetes practice in India, there was a mixed opinion among these doctors. A total of 90 $(56.25 \%)$ doctors with BHMS/BAMS/BUMS degree believe that MBBS degree is enough to do a legal diabetes practice in India. This indicates lack of awareness among these doctors regarding diabetes practice. Whereas only $40 \%$ of MBBS doctors believe that at least MD degree is required to do a legal diabetes practice in India. Other responses which were recorded are shown in Figure 2.

When asked whether the needs of diabetic population in rural India are properly addressed, majority of doctors from both the fields had common opinion i.e. NO (Figure 3 ), but the reason they stated was different among them 
(Figure 4). Almost 82\% of BHMS/BAMS/BUMS doctors believe the reason was lack of qualified doctors whereas up to $40 \%$ of MBBS doctors believed the reason was lack of awareness among the rural patients. Whatever opinion the doctors have, it is a known fact that the needs of diabetics' are not addressed by the doctor community, especially in rural India.

\section{OPINION OF DOCTORS ABOUT TIIY UNMET NEKDS OF RIRAL, DLABETICS}

Indiu is home to nearly 62 million dabetics - second only w China which has over 92 million diabetics. Although the prevaleasce of diabetes is lower in rual lindia, the wotal sumber of diabetic patents in runl anes is much higher, since over $70 \%$ of the population in India fries in rural areas. The present study is to explore the opinion of varives doctors about the unnet needs of diabetes in nural ladia.

1. What is your qualification?

DBHMSBAMSBUMS

UMBBS

2. What is the minimum degree requind legally to do a diabetes practice in India? DDochors with BHMSBAMSBOMS degrec

DDockars with at least MBRS degree

DMBRS Dockars with some fellowslip courses in diateles

DDochors with MD - General Medicine JDM-Endocrinology degrex

3. Doyou think the neats of diabetic population in rural india are peoperily addinessod?

पYes

口No

4. IfNo, what might be the reava? (If yes to the previrus question then skip this question)

Dlakk of awaratess among the runal patients

Olnedequate health care resources in rusal arcas

Disck of qualified doctoos

DAmy other reason, please mention.

5. Who will be at a better position to provide peeveative and basichrimary care for diabebes with proper referals and provide awanenes programmes at community level in nural ladia?

DDochors with BHMS / BAMS degree

DDockars with MBRS

DMBBS Doctors with some fellowsip degrox in diabctes

DDoctars with MD - General degreed DM- Endocrinalogy degree

6. Do you think that doctors with allied degross like BHMS, BAMS and BUMS are compckent cnough to manage diabctes?

पYes

【No

7. Do you think MBBS doctors with sone fellowship courses in Diabetes can manage disbetes effectively (exocpt setivus complications)?

DYes

¿No

8. Whether MCI recognizes icllowship courses in dialetology?

¿Yes

¿No

Figure 1: Pretested questionnaire.

When asked, who will be at a better position to provide preventive and basic/primary care for diabetics with proper referrals and provide awareness programmes at community level in rural India? Majority of BHMS/BAMS/BUMS doctors $(68.75 \%)$ believed that their profession can do that job well whereas doctors with MBBS degree $(63.15 \%)$ believe that only MBBS doctors can do that job better (provided they are supported by basic facilities and good pay). Other opinions among these doctors are shown in Figure 5.

When asked regarding the competency of doctors with allied degrees like BHMS, BAMS and BUMS in managing diabetes, BHMS/BAMS/BUMS doctors $(87.5 \%)$ had a firm opinion that they were competent enough, whereas, $94.74 \%$ of MBBS doctors believe that BHMS/BAMS/BUMS doctors were not competent to do a diabetes practice.

When asked whether MBBS doctors with some fellowship courses in diabetes can manage diabetes effectively (except serious complications), doctors from both the fields had a positive opinion.

When asked whether MCI recognizes fellowship courses in diabetology? majority of BHMS/BAMS/BUMS doctors $(93.75 \%)$ believed that $\mathrm{MCI}$ recognizes these fellowship courses but $58 \%$ of MBBS doctors believed that MCI doesn't recognized these fellowship courses.

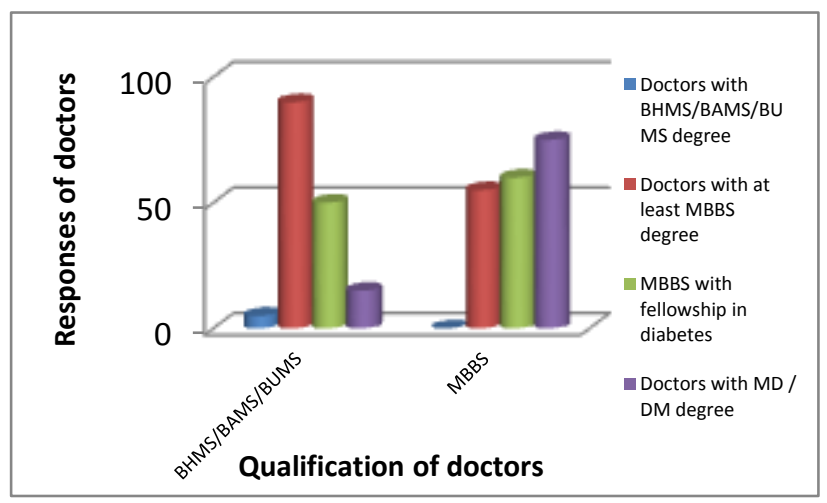

Figure 2: Responses from doctors to the question "what is the minimum degree required legally to do a diabetes practice in India".

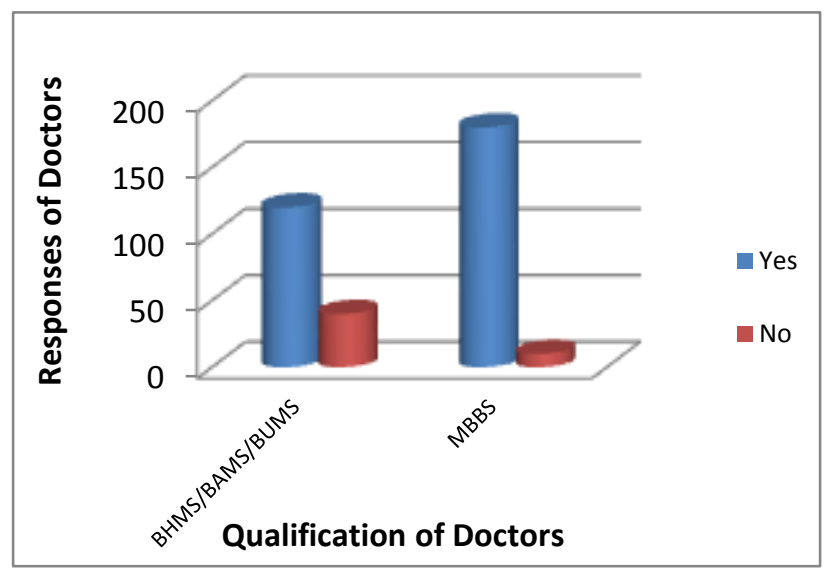

Figure 3: Responses from doctors to the question "Do you think the needs of diabetic population in rural India are properly addressed?". 


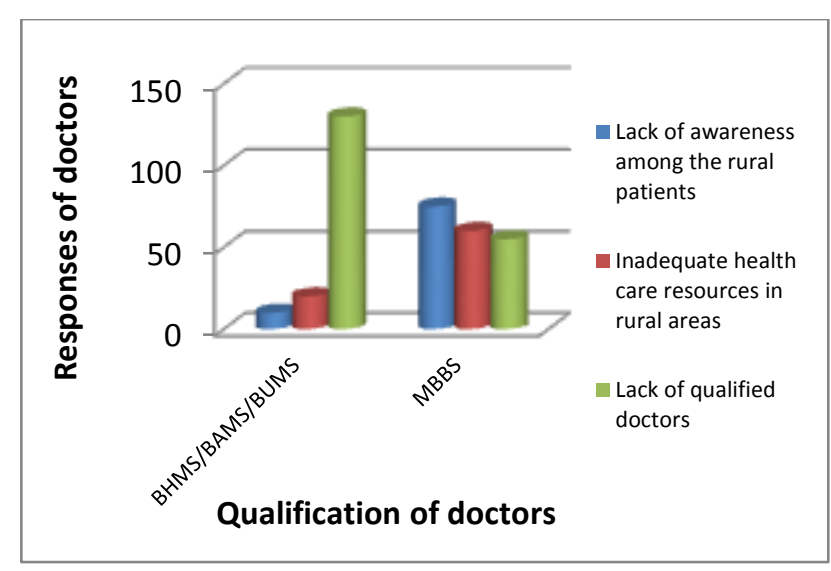

Figure 4: Responses from doctors to the question "Reasons behind not addressing the needs of rural diabetics".

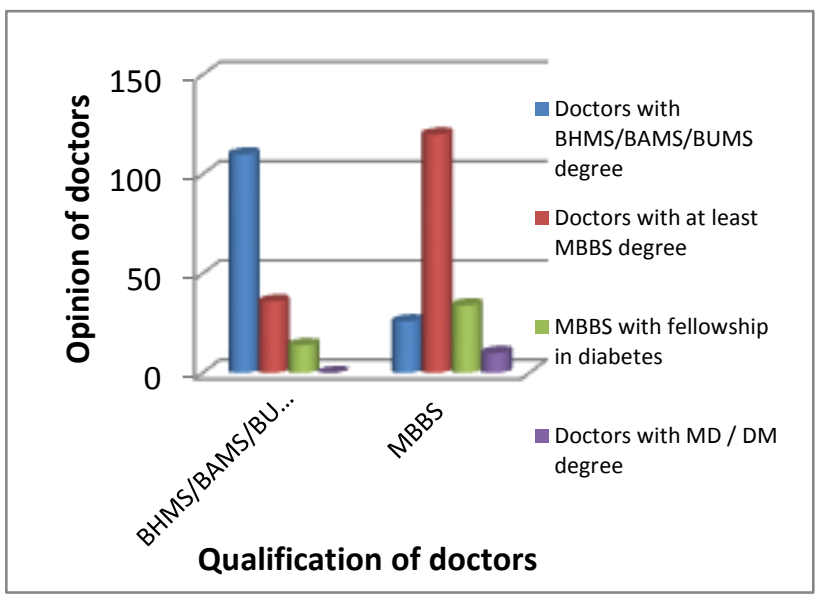

Figure 5: Responses from doctors to the question "Who will be at a better position to provide preventive and basic/primary care for diabetics with proper referrals and provide awareness programmes at community level in rural India".

\section{DISCUSSION}

After recording the responses it was clear that doctors had differing opinion about the diabetes practice. Awareness is also low among few doctors. In India only MD (general medicine) and endocrinologists are technically qualified to treat diabetes. A lot of doctors / physicians who are interested in diabetes management and have the skills to manage diabetes fail to do justice to their patients because of so many hurdles. Physicians / doctors cannot legally do a diabetes practice without a valid degree. Doctors cannot afford a MD/DM degree (Either because of competition or financial constraints) to start a diabetes practice. So they opt for some fellowship degrees offered by numerous institutes in India. None of these degrees are approved by MCI. These doctors still manage their practice and claim themselves to be diabetologist after obtaining these fellowship/diploma degrees. In Andhra Pradesh alone around 600 MBBS graduates are portraying themselves as diabetologists and it is in violation of the Medical Council of India (MCI) regulations. But diabetic populations' need cannot be met by the true endocrinologist or MD General Medicine doctors alone, since their number is very low compared to the rising number of diabetics. One of the endocrinologist has to say that since diabetes is not a difficult disease to treat and its medicines are not as harmful or toxic as cancer drugs, MBBS doctors seem to get away without any problems most of the times. ${ }^{14}$

\section{CONCLUSION}

All the doctors who were involved in the study had different opinion. MBBS doctors are more aware than BHMS/BAMS/BUMS doctors about the minimum degree required doing a diabetic practice legally in India. Both the group of doctors believed that the needs of diabetic population in rural India are not properly addressed; the reason behind this was lack of qualified doctors (according to majority of BHMS/BAMS/BUMS doctors) and lack of awareness among the rural patients (according to MBBS doctors). Both the doctors believe that MBBS doctors with some fellowship courses in diabetes can manage diabetes effectively (except serious complications). MCI should come forward and recognize these fellowship courses by regularizing them with proper guidelines and protocol set up for the institution providing such courses and allow those doctors to practice in rural areas.

Funding: No funding sources Conflict of interest: None declared

Ethical approval: The study was approved by the Institutional Ethics Committee

\section{REFERENCES}

1. Guariguata L. Whiting DR, Hambleton I, Beagley J, Linnenkamp U, Shaw JE. Global estimates of diabetes prevalence for 2013 and projections for 2035. Diabetes Res Clin Pract. 2013;103(2):137-49.

2. International Diabetes Federation 2015. Available at http://www.idf.org/files/idf_publications/idf_annual _report_2012_EN/index.html. Accessed 11 October 2015.

3. Todd Cade W. diabetes-related microvascular and macrovascular diseases in the physical therapy setting. Phys Ther. 2008;88(11):1322-35.

4. Indiatimes.com. Available from http://timesofindia.indiatimes.com/life-style/healthfitness/health/Diabetes-can-be-checked-amongmost-Indians/articleshow/29905164.cms. Accessed 11 October 2015.

5. Ramachandran A. Socio-economic burden of diabetes in India. JAPI. 2007;55:9-12.

6. Tuso P. Prediabetes and lifestyle modification: time to prevent a preventable disease. Perm J. 2014;18(3): 88-93. 
7. American diabetes association. Nutrition recommendations and interventions for diabetes. Diabetes Care. 2007;30(1):S48-65.

8. Mohan V. Tele-diabetology to screen for diabetes and associated complications in rural India. J Diabetes Sci Technol. 2014;8(2):256-1.

9. Oputa RN, Chinenye S. Diabetes mellitus: A global epidemic with potential solutions. African Journal of Diabetes Medicine. 2012;20(2):33-5.

10. Jonas JB. Diabetes mellitus in rural India. Epidemiology. 2011;22(1):134-5.

11. Indiatimes.com. Available from http://timesofindia.indiatimes.com/city/pune/Studyfinds-diabetes-in-rural youths/articleshow/38883918.cms. Accessed 11 October 2015.
12. Mohan V, Pradeepa. Epidemiology of diabetes in different regions of India. Health Administrator. 2009;22(1):1-18.

13. Joshi SR, Das AK, Vijay VJ, Mohan V. Challenges in diabetes care in India: sheer numbers, lack of awareness and inadequate control. J Assoc Physicians India. 2008;56:443-50.

14. Indiatimes.com. Available from http://timesofindia.indiatimes.com/city/hyderabad/D iabetologists-under-govtscanner/articleshow/20564812.cm. Accessed 11 October 2015.

Cite this article as: Dixit R, Pokala N. Opinion of doctors about unmet needs of rural diabetics. Int $\mathrm{J}$ Basic Clin Pharmacol 2016;5:116-20. 